\title{
Pacific
}

Journal of

Mathematics

\section{PERIODIC POINTS ON NILMANIFOLDS AND SOLVMANIFOLDS}

\author{
EDWARD KEPPELMANN
}




\title{
PERIODIC POINTS ON NILMANIFOLDS AND SOLVMANIFOLDS
}

\author{
Edward Charles KeppelmanN
}

Let $M$ be a compact manifold and $f: M \rightarrow M$ a self map on $M$. For any natural number $n$, the $n$th iterate of $f$ is the $n$-fold composition $f^{n}: M \rightarrow M$. The fixed point set of $f$ is $\operatorname{fix}(f)=$ $\{x \in M: f(x)=x\}$. We say that $x \in M$ is a periodic point of $f$ if $x$ is a fixed point of some $f^{n}$ and we denote the set of all periodic points of $f$ by $\operatorname{per}(f)=\bigcup_{n=1}^{\infty} f \operatorname{ix}\left(f^{n}\right)$. A periodic point $x$ of $f$ is said to have minimal period $k$ provided that $k$ is the smallest integer for which $x \in \operatorname{fix}\left(f^{k}\right)$. We say that $\operatorname{per}(f)$ is homotopically finite, denoted $\operatorname{per}(f) \sim$ finite, iff there is a $g$ homotopic to $f$ such that $\operatorname{per}(g)$ is finite. When $M$ is a torus B. Halpern has shown that $\operatorname{per}(f) \sim$ finite iff the sequence of Nielsen numbers $\left\{N\left(f^{n}\right)\right\}_{n=1}^{\infty}$ is bounded. The main objective of this work is to extend these results to all nilmanifolds and to consider to what extent they can be extended for compact solvmanifolds. A compact nilmanifold is a coset space of the form $M=G / \Gamma$ where $G$ is a connected, simply connected nilpotent Lie group and $\Gamma$ is a discrete torsion free uniform subgroup. For these spaces we have the additional result that when the homotopy can be accomplished, the resulting $g$ satisfies $\left|f \operatorname{xx}\left(g^{n}\right)\right|=N\left(f^{n}\right)$ for all $n$ with $N\left(f^{n}\right) \neq 0$ and if $\left\{N\left(f^{n}\right)\right\}_{n=1}^{\infty}=\{0\}$ then we can choose $g$ to be periodic point free. Also, when $f$ is induced by a homomorphism $F: G \rightarrow G$, then we can write $g=u f v$ where $u$ and $v$ are isotopic to the identity. This form for the homotopy is used to find sufficient conditions for $\operatorname{per}(f) \sim$ finite when $M$ is a solvmanifold. We then present a model for a specific class of solvmanifolds where these conditions can be considered. This allows us to prove the general result in a variety of low dimensional examples.

Consider two self maps $f$ and $g$ on $M$. Classical Nielsen fixed point theory concerns itself with the study of how $\operatorname{fix}(f)$ and $\operatorname{fix}(g)$ are related when $g$ is homotopic to $f$, written $g \sim f$. In particular, the Nielsen number $N(f)$ of $f$ is a lower bound on the number of fixed points of any map in the homotopy class of $f$. The Nielsen number $N(f)$ is defined by partitioning $f x(f)$ into equivalences classes (called Nielsen classes) and assigning an integer index to each class. Points $x, y \in \operatorname{fix}(f)$ are said to be Nielsen equivalent, denoted $x \sim_{f} y$ provided that there is a path $\omega$ from $x$ to $y$ in which (rel endpoints) $\omega \sim f \omega$. For a smooth map and isolated fixed points the fixed point 
index of a Nielsen class is simply

$$
\sum_{y \sim \sim_{f} x} \operatorname{sgn}\left[\operatorname{det}\left(D f_{y}-I\right)\right] .
$$

$N(f)$, a homotopy invariant of $f$, is then the number of essential Nielsen classes (i.e. those with nonzero index) of $f$ [14]. A general construction for finding a $g \sim f$ with exactly $N(f)$ fixed points is available in many circumstances (see $[6,13]) . N(f)$ can often be calculated by various algebraic invariants of the map $f$ (see [14]).

When $g \sim f$, then it follows that for any value of $n, g^{n} \sim f^{n}$. It is not true, however, that if $h, f: X \rightarrow X$ are such that $h^{n} \sim f^{n}$ for some $n$, that the homotopy can always be realized as a deformation of $h$ to $g$ (unless of course $n=1$ ). Thus, in general, for various values of $n$, we have that

$$
\min \left\{\left|\operatorname{fix}\left(g^{n}\right)\right|: g \sim f\right\} \geq \min \left\{|\operatorname{fix}(h)|: h \sim f^{n}\right\} \geq N\left(f^{n}\right) .
$$

There is a well developed Nielsen theory for periodic points $[10,11]$ which concerns itself with determining, for a fixed $n$, the minimum values for $\left|\operatorname{fix}\left(g^{n}\right)\right|$ and $\mid\left\{x \in \operatorname{fix}\left(g^{n}\right): x\right.$ has minimal period $\left.n\right\} \mid$ as $g$ varies over all maps homotopic to $f$. The nature of this work is different, in two important ways, from these general studies. Here our focus is completely on the class of compact solvmanifolds (of which compact nilmanifolds are an important part), and we are concerned with the size of $\operatorname{per}(g)$ for $g \sim f$. That is, we want to know as a whole (for all iterates simultaneously), how the periodic point set of a map can be changed under homotopy.

We begin our study with $\S 1$ where Halpern's results for tori are reviewed and reformulated for our use. Section 2 is an extension of Halpern's results to nilmanifolds. Section 3 is then an examination of the most general extension of these methods to the class of compact solvmanifolds. Finally, by specializing to a common class of special solvmanifolds in $\S 3.1$ we can then remove the extra hypothesis of our general results and prove theorems much like Halpern's in dimensions 3 or less.

1. Periodic points on tori. Let $\mathbf{R}$ denote the real numbers and $\mathbf{Z}$ the integers and for a fixed natural number $m$ consider the quotient space $\mathbf{R}^{m} / \mathbf{Z}^{m}$. Now if the euclidean space $\mathbf{R}^{m}$ is given the usual additive group structure then this quotient is the $m$-dimensional torus $T^{m}$. If, however, $\mathbf{R}^{m}$ is thought of as a nilpotent Lie group (say a subgroup of some multiplicative group of unit upper triangular matrices), then 
the quotient is a typical nilmanifold $M^{m}$. Finally, if $\mathbf{R}^{m}$ is endowed with the structure of a solvable Lie group then we have an example of a special solvmanifold $R^{m}$. (The general solvmanifold is the quotient of a connected, simply connected, solvable Lie group by a uniform (not necessarily discrete) subgroup [17].) We begin our study of periodic points on this hierarchy of spaces by first considering the tori as was done by Halpern in [8].

From the standard theory for aspherical spaces, we know that any map $f: T^{m} \rightarrow T^{m}$ is up to homotopy, covered by a linear map $F: \mathbf{R}^{m} \rightarrow \mathbf{R}^{m}$ whose matrix with respect to the standard basis of $\mathbf{R}^{m}$ has integer entries. It can be shown that

THEOREM $1.1[5,8]$. Suppose $f: T^{m} \rightarrow T^{m}$ is covered by a linear map $F: \mathbf{R}^{m} \rightarrow \mathbf{R}^{m}$. For every natural number $n$

$$
N\left(f^{n}\right)=\left|L\left(f^{n}\right)\right|=\left|\operatorname{det}\left(I-F^{n}\right)\right|=\prod_{i=1}^{m}\left|1-\alpha_{i}^{n}\right|
$$

where the complex numbers $\alpha_{1}, \ldots, \alpha_{m}$ are the eigenvalues, with multiplicity, of $F$. Furthermore, if $\left|L\left(f^{n}\right)\right| \neq 0$ then the fixed points of $f^{n}$ are isolated and all belong to distinct Nielsen classes with the same index. Thus $\left|\mathrm{fix}\left(f^{n}\right)\right|=N\left(f^{n}\right)$.

We begin by considering a certain kind of homotopy which will be of great use to us.

Definition 1.2. Deformants and deformant homotopies. Suppose $f, g: X \rightarrow Y$. We say that $g$ is a deformant homotopy of $f$ if we can write $g=u f v$ where $u$ and $v$ are isotopic to the identity on $X$ or $Y . u$ and $v$ are called, respectively, the left and right deformants. $\mathscr{D}(X)$ will denote the set of all deformants on $X$. If $u=\mathrm{id}$ this is a right-deformant homotopy whereas if $v=\mathrm{id}$ we call this a left-deformant homotopy. The general situation with both $u$ and $v$ nontrivial is called a bi-deformant of $f$. We use $g \sim_{D} f$ to denote that $g$ is a deformant homotopy (left, right, or bi) of $f$. It is not difficult to see that the relation of deformant homotopy between self maps on a space $X$ is an equivalence relation. The most common example of a deformant for Halpern's results is that induced by a flow $\left\{F_{t}: X \rightarrow X\right\}_{t \in \mathbf{R}}$.

In what follows we assume as in Theorem 1.1 that $f: T^{m} \rightarrow T^{m}$ is induced by a linear map $F$ on $\mathbf{R}^{m}$. Because the eigenvalues of $F$ are those of a matrix with integer entries, Halpern [8] was able to 
show the following regarding the sequence of Nielsen numbers of the iterates of $f$.

LEMMA 1.3. Let $f: T^{m} \rightarrow T^{m}$. As a function of $n, N\left(f^{n}\right)=$ $\left|L\left(f^{n}\right)\right|=\prod_{i=1}^{m}\left|1-\alpha_{i}^{n}\right|$ is either identically zero (iff some $\alpha_{i}=1$ ) or is not identically zero and is bounded iff $\forall i \quad\left|\alpha_{i}\right| \leq 1$. In the latter case, each nonzero $\alpha_{i}$ is a root of unity.

Suppose that $f: X \rightarrow X$ is such that some iterate $f^{n}$ of $f$ is the identity on $X$. Then clearly $\left\{N\left(f^{n}\right)\right\}_{n=1}^{\infty}$ will also be periodic and hence bounded. In the case of the torus, it turns out that the ability to deform certain periodic maps to have a finite periodic point set with the desired properties allows one to do the same for all maps with a bounded sequence of Nielsen numbers. The following result provides us with a means of doing this.

THEOREM 1.4 [8]. Let $M^{m}$ be a smooth compact manifold of dimension $m \geq 2$. Suppose $f: M^{m} \rightarrow M^{m}$ is such that, for some $N \geq 2$, $f^{N}=\mathrm{id}_{M^{m}}$. Then if $f$ has a nonempty fixed point set and the subset $P$ of $M^{m}$ consisting of those points with minimal period less than $N$ is finite, then there is a smooth $g: M^{m} \rightarrow M^{m}$ such that $g \sim_{D} f$ by either a left or right deformant homotopy, $g|\mathbb{P}=f| P$, and $P=\operatorname{per}(g)$. Furthermore, in the case of $m=1$, we can allow $M^{1}$ to be the circle $S^{1}$ and $f$ to be either the identity or the standard map of degree -1 . In the former case a left deformant of $f$ is periodic point free while in the latter case a left deformant of $f$ has exactly two periodic points, both of which are fixed points.

This leads directly to our major result for tori. The main theorem is from [8] but our observations about the role of deformant homotopies is essential for later sections.

Theorem 1.5 (Periodic points on tori). Suppose $f: T^{m} \rightarrow T^{m}$ is such that $\left\{N\left(f^{n}\right)\right\}_{n=1}^{\infty}=\left\{\left|L\left(f^{n}\right)\right|\right\}_{n=1}^{\infty}$ is bounded. Then there is a $g \sim f$ with $\operatorname{per}(g)$ finite so that, for each $n=1,2 \ldots, L\left(f^{n}\right) \neq$ $0 \Rightarrow\left|\operatorname{fix}\left(g^{n}\right)\right|=N\left(f^{n}\right)$. If $\left\{N\left(f^{n}\right)\right\}_{n=1}^{\infty}=\{0\}$ then $g$ is periodic point free. If $f$ is induced by a linear map on $\mathbf{R}^{m}$ then $g \sim_{D} f$ by a left deformant homotopy.

For our work the correct point of view for the proof of the above theorem is illustrated by the following corollary. Recall that a partition 
of a positive integer $m$ is a finite sequence $\mathscr{A}=m_{1}, \ldots, m_{k}$ of positive integers whose sum is $m$. A induces a decomposition of $\mathbf{R}^{m}\left(T^{m}\right)$ into a $k$-fold product in which the $i$ th factor is $\mathbf{R}^{m_{i}}\left(T^{m_{i}}\right)$.

Corollary 1.6. Suppose $f: T^{m} \rightarrow T^{m}$ is induced by a linear map $F: \mathbf{R}^{m} \rightarrow \mathbf{R}^{m}$ with $\left\{N\left(f^{n}\right)\right\}_{n=1}^{\infty}$ bounded. Let $g$ be the result of applying Theorem 1.5 to $f$. Let $r=\operatorname{dim} \operatorname{Ker}(F)$. Let $\mathscr{A}=m_{1}, \ldots, m_{k}$ be a partition of $m-r$ so that $m_{i}$ is the number of eigenvalues of $F$, listed by algebraic multiplicity, which are primitive $b_{i}$ th roots of unity where $b_{1}<b_{2}<\cdots<b_{k}$. Then there are maps $g_{1}, \ldots, g_{k}, h$ with $g_{i}: T^{m_{i}} \rightarrow T^{m_{i}}$ and $h: T^{r} \rightarrow T^{r}$ so that:

1. $h$ is constant. Thus $\operatorname{per}(h)$ is a singleton.

2. For each $i, g_{i}$ is the result of applying Theorem 1.4 to a periodic map on $T^{m_{i}}$ of period $b_{i}$. Thus $\operatorname{per}\left(g_{i}\right)$ is finite and all elements of $\operatorname{per}\left(g_{i}\right)$ have a minimal period which is less than $b_{i}$ and divides $b_{i}$.

3. Modulo a period preserving change of coordinates conjugacy $\operatorname{per}(g)=\operatorname{per}\left(g_{1} \times \cdots \times g_{k} \times h\right)$ and thus $\operatorname{per}(f) \sim$ finite.

4. If $b_{1}=1$, then $\operatorname{per}\left(g_{1}\right)=\varnothing$ so $\operatorname{per}(g)=\varnothing$.

Using Corollary 1.6 we can now do our own analysis on the nature of the periodic point set that results when Theorem 1.5 is applied to a map. This analysis will be especially useful for our later work on solvmanifolds. We will first introduce some terminology.

Definition 1.7. Inessential periodic points. Suppose $f: X \rightarrow X$ is a map on a space where $L(f)$ is defined. We say that $x \in \operatorname{per}(f)$ is an inessential periodic point of $f$ provided that the minimal period $m_{x}$ of $x$ satisfies $L\left(f^{m_{x}}\right)=0$.

By Theorem 1.5 we know that any self map $h$ on a torus with $L(h)=0$ can be deformed to be fixed point free. Thus we are using the term inessential to mean that $f^{m_{x}}$ can be deformed to remove (or make empty) all the Nielsen classes of $f^{m_{x}}$. It is an open question of some interest to decide in general if inessential really means that we can deform $f$ to remove all such periodic points. In the case of very low dimensional tori we can indeed prove such a result from Corollary 1.6 merely by analyzing the possible eigenvalues which can occur in a $2 \times 2$ matrix with integer entries whose nonzero eigenvalues are roots of unity.

Corollary 1.8. Suppose $f: T^{m} \rightarrow T^{m}$ is induced by a linear map $F: \mathbf{R}^{m} \rightarrow \mathbf{R}^{m}$ and $\left\{N\left(f^{n}\right)\right\}_{n=1}^{\infty}$ is bounded. If $m=1$ or 2 , then 
the result of applying Theorem 1.5 to $f$ is a map $g \sim f$ with no inessential periodic points.

It turns out that there are certain situations when for arbitrary $m$ and $f: T^{m} \rightarrow T^{m}$ with $\left\{N\left(f^{n}\right)\right\}_{n=1}^{\infty}$ bounded we can say that the deformation of Theorem 1.5 produces a map with no inessential periodic points. We now explore the theory which depicts these conditions.

Definition 1.9. Toroidal divisor multiples. For two positive integers $r$ and $s$ let $r \mid s$ denote that $r$ divides $s$. Let $\mathscr{B}=\left\{d_{1}, \ldots, d_{p}\right\}$ be a finite set of positive integers. A toroidal divisor multiple for $\mathscr{B}$ is a set $\left\{l_{1}, \ldots, l_{p}\right\}$ of positive integers such that for each $i l_{i}$ divides $d_{i}$ and there is a $d_{j}$ which divides the least common multiple of $\mathscr{B}$.

Theorem 1.10. Suppose $f: T^{m} \rightarrow T^{m}$ is induced by a linear map $F: \mathbf{R}^{m} \rightarrow \mathbf{R}^{m}$ and $\left\{L\left(f^{n}\right)\right\}_{n=1}^{\infty}$ is bounded. Let $\mathscr{B}=\left\{b_{1}, \ldots, b_{k}\right\}$ be the set arising from Corollary 1.6. If $1 \in \mathscr{B}$ or $\mathscr{B}$ has no toroidal divisor multiples then we can assume that the deformation of Theorem 1.5 applied to $f$ produces a map with no inessential periodic points.

Unlike the situation on $T^{2}$, maps on $T^{3}$ can lead to toroidal divisor multiples and, despite Halpern's methods, inessential periodic points.

2. Nilmanifolds. Our goal in this section is to extend Halpern's results for tori to the case of nilmanifolds. We will start with a quick overview of the definitions and basic properties of nilmanifolds. This will allow us to see the analogies between nilmanifolds and tori as well as the new features which these spaces have that set the scene for the results which follow.

A nilmanifold is a smooth compact manifold on which a nilpotent Lie group acts smoothly and transitively. The following result is a fundamental characterization of nilmanifolds. Recall that a subgroup $\Gamma$ of a topological group $G$ is called uniform provided that $G / \Gamma$ is compact.

THEOREM 2.1 [15]. Every nilmanifold $M^{m}$ is equivariantly homeomorphic to a left coset space of the form $G / \Gamma$ where $G \approx \mathbf{R}^{m}$ is a connected, simply connected nilpotent Lie group and $\Gamma \approx \mathbf{Z}^{m}$ is a uniform discrete torsion free subgroup.

Thus $\mathbf{R}^{m}$ is the universal covering space of every nilmanifold of dimension $m$. Furthermore, $M^{m}$ is aspherical with $\pi_{1}\left(M^{m}\right) \cong \Gamma$. 
A fundamental result $[15,7]$ shows that any endomorphism on $\Gamma$ extends uniquely to a Lie group endomorphism on $G$. Thus as in the case of the tori we see that the homotopy classes of self maps of a nilmanifold $M^{m}$ are in one-to-one correspondence with the set of endomorphisms of $G$ which are invariant on $\Gamma$. Thus, up to homotopy, we can assume that any self map $f: M^{m} \rightarrow M^{m}$ is induced by a homomorphism $F: G \rightarrow G$. The descending central series for $G$ is given by $G_{0}=G$ and, for $i>0, G_{i}=\left[G, G_{i-1}\right]$. The nilpotence of $G$ means that, for some maximal value of $k, G_{k} \neq 0$. We let $\widetilde{G}=G_{k}$. It is then clear that $\widetilde{G}$ is an abelian $F$ invariant subgroup of $G$ so the following can be proved:

Theorem $2.2[7,2]$. Suppose $g: M^{m}=G / \Gamma \rightarrow M^{m}$ is a map on a nilmanifold. (We assume that $G$ is nonabelian so that $M^{m}$ is not a torus.) Then, up to homotopy, $g$ can be replaced by a map $f: M^{m} \rightarrow M^{m}$ which is induced by a homomorphism $F: G \rightarrow G$. $F$ induces homomorphisms $F_{0}: \widetilde{G} \rightarrow \widetilde{G}, \bar{F}: G / \widetilde{G} \rightarrow G / \widetilde{G}$, and, by taking derivatives, Lie algebra homomorphisms $\dot{F}: \mathscr{G} \rightarrow \mathscr{G}, \dot{F}_{0}: \widetilde{\mathscr{G}} \rightarrow$ $\widetilde{\mathscr{G}}$, and $\dot{\bar{F}}: \overline{\mathscr{G}} \rightarrow \overline{\mathscr{G}}$. Thus $F$ induces maps $f_{0}: \widetilde{G} / \widetilde{\Gamma} \cong T^{r} \rightarrow T^{r}$, $f: G / \Gamma=M^{m} \rightarrow M^{m}$, and $\bar{f}:(G / \widetilde{G}) /(\Gamma / \widetilde{\Gamma}) \cong B^{s} \rightarrow B^{s}$ which give a fiber preserving map of the Fadell-Husseini fibration $T^{r} \hookrightarrow M^{m} \rightarrow B^{s}$ of $M^{m}$. Here $B^{s}$ is a nilmanifold of dimension $s$ and $T^{r}$ is a torus of dimension $r$ with $m=s+r$. For each $n$ we have that

$$
\begin{aligned}
L\left(f^{n}\right) & =L\left(f_{0}^{n}\right) L\left(\bar{f}^{n}\right)=\operatorname{det}\left(I-\dot{F}_{0}^{n}\right) \operatorname{det}\left(I-\dot{\bar{F}}^{n}\right) \\
& =\prod_{i=1}^{r}\left(1-\alpha_{i}^{n}\right) \prod_{j=1}^{s}\left(1-\beta_{j}^{n}\right)=\operatorname{det}\left(I-\dot{F}^{n}\right)
\end{aligned}
$$

where $\alpha_{1}, \ldots, \alpha_{r}, \beta_{1}, \ldots, \beta_{s}$ are the eigenvalues, listed with their multiplicities, of $\dot{F}$ and $\dot{F}_{0}$, respectively. Therefore, in regard to Nielsen numbers we have that

$$
|L(f)|=\left|L\left(f_{0}\right)\right||L(\bar{f})|=N\left(f_{0}\right) N(\bar{f})=N(f) .
$$

Let $F \hookrightarrow E \rightarrow B$ be a Hurewicz fibration of compact manifolds with projection $\pi: E \rightarrow B$. For $x \in B, F_{x}=\pi^{-1}(x)$ is the fiber over $x$. If $f: E \rightarrow E$ is a fiber preserving map then the map on the fiber over $x$ of $f$, denoted $f_{x}$, is the restriction $f \mid \pi^{-1}(x): F_{x} \rightarrow F_{\bar{f}(x)}$. Hurewicz fibrations are equipped with a path lifting function. For each path $\omega$ in $B$ this gives rise to a fiber translation map $\tau_{\omega}: F_{\omega(0)} \rightarrow$ $F_{\omega(1)}$. The coset structure of our Fadell-Husseini fibration and the fact 
that $f$ is induced by a homomorphism means that we can take our lifting function to satisfy the following.

LEMMA 2.3. If $f: M^{m}=G / \Gamma \rightarrow M^{m}$ is induced by a homomorphism $F: G \rightarrow G$ then $\tau_{\bar{f} \omega} f=f \tau_{\omega}$.

Anosov's original proof of the fact that $|L(f)|=N(f)$ on a nilmanifold was done independently of the Fadell-Husseini proof and did not use the idea of the Fadell-Husseini fibration. Anosov's argument was that all essential Nielsen classes have the same index \pm 1 . The result then follows from the Lefschetz index theorem once it is shown that whenever $L(f)=0$ then $f$ is homotopic to a fixed point free map. It is Anosov's proof of this last fact that gives us the following lemma which we will exploit to make $f$ periodic point free.

TheOREM 2.4 [2] (The Anosov trick). Assume that $f: M^{m}=G / \Gamma \rightarrow$ $M^{m}$ is a self map on a nilmanifold which is induced by a homomorphism $F: G \rightarrow G$. Furthermore, suppose $0 \neq Y \in \widetilde{\mathscr{G}}$ is such that $Y \notin(\dot{F}-I)(\mathscr{G})$. For each $t \in \mathbf{R}$ define $f_{t}: N \rightarrow N$ by $f_{t}(x)=\exp (t Y) f(x)$. Then the set $\mathscr{A}=\left\{t \in \mathbf{R}: \operatorname{fix}\left(f_{t}\right) \neq \varnothing\right\}$ is at most countable.

Periodic points on nilmanifolds. We can now extend Halpern's results to nilmanifolds. In doing so we will need the following easy fact.

LEMMA 2.5 (Periodic points of compositions). Let $X_{0}, \ldots, X_{p-1}$ be topological spaces and $f_{0}, \ldots, f_{p-1}$ be maps with $f_{i}: X_{i} \rightarrow X_{i+1}(i+1$ computed $\bmod p)$. Let, for $j=0,1, \ldots, p-1, g_{j}=f_{j-1} \cdots f_{0} f_{p-1}$ $\cdots f_{j+1} f_{j}: X_{j} \rightarrow X_{j}$. Then for each $j$ and $k$ there is a 1-1 correspondence between $\operatorname{per}\left(g_{j}\right)$ and $\operatorname{per}\left(g_{k}\right)$. In particular, if $\operatorname{per}\left(g_{0}\right)$ is finite then so are all the $\operatorname{per}\left(g_{i}\right)$.

THEOREM 2.6 (Periodic points on nilmanifolds). Suppose $f: M^{m}=$ $G / \Gamma \rightarrow M^{m}$ is a map on a nilmanifold with $\left\{N\left(f^{n}\right)\right\}_{n=1}^{\infty}=\left\{\left|L\left(f^{n}\right)\right|\right\}_{n=1}^{\infty}$ bounded. Then there is a $g \sim f$ with $\operatorname{per}(g)$ finite so that, for each $n=1,2, \ldots, L\left(f^{n}\right) \neq 0 \Rightarrow\left|\operatorname{fix}\left(g^{n}\right)\right|=N\left(f^{n}\right)$. If $\left\{N\left(f^{n}\right)\right\}_{n=1}^{\infty}=\{0\}$ then $g$ is periodic point free. If $f$ is induced by a homomorphism $F: G \rightarrow G$ then we have that $g \sim_{D} f$.

Proof. The result is proved by induction on $m$. Since the only nilmanifolds of dimensions 1 and 2 are the circle and 2-torus respec- 
tively, Theorem 1.5 gives us the result for these $m$. We now allow $m$ to be arbitrary and assume the theorem for all dimensions $<m$. Assuming that $f$ is induced by a homomorphism means from the definition of the Fadell-Husseini fibration that $\bar{f}$ is also induced by homomorphism. The setup of Theorem 2.2 thus applies with $\left\{L\left(f^{n}\right)\right\}_{n=1}^{\infty}$ bounded. We show how to produce isotopies $u$ and $v$ of $\mathrm{id}_{M^{m}}$ so that $u f v$ has the desired periodic point set by considering two cases based on whether or not $\left\{L\left(\bar{f}^{n}\right)\right\}_{n=1}^{\infty}$ is bounded.

Case 1. $\left\{L\left(\bar{f}^{n}\right)\right\}_{n=1}^{\infty}$ bounded. If $\left\{L\left(\bar{f}^{n}\right)\right\}_{n=1}^{\infty}$ is bounded then we may use the induction hypothesis to get $\bar{u}, \bar{v} \sim^{h} \operatorname{id}_{B}$ with $\operatorname{per}(\bar{u} \bar{f} \bar{v})$ finite. Since homotopies in a fibration can be lifted using the fiber translation maps induced from the Hurewicz lifting function, $\bar{u}$ and $\bar{v}$ can thus be lifted to fiber-preserving deformants $u$ and $v$ over $\bar{u}$ and $\bar{v}$, respectively. Thus $f \sim_{D} u f v$ and $u f v$ will be fiber preserving with finitely many periodic fibers. Now if $L(f)=L(\bar{f}) L\left(f_{0}\right)=0$ and it is because $L(\bar{f})=0$ then we can assume $\bar{u}$ and $\bar{v}$ are chosen so that $\operatorname{per}(\bar{u} \bar{f} \bar{v})=\varnothing$ so necessarily $\operatorname{per}(u f v)=\varnothing$ and we are done. Otherwise, we must show how to deform the maps on the periodic fibers of $u f v$ (using deformants) to obtain that the appropriate iterates of these maps on fibers each have a finite periodic point set. These deformants of maps on fibers can then be combined and extended to a global deformant over $\mathrm{id}_{B^{s}}$ using the local triviality of the fibration. Thus our resulting deformant homotopy of $f$ will be a fiber preserving map $g$ with projection $\bar{u} \bar{f} \bar{v}$. Thus no new periodic fibers are introduced during these fiber map homotopies and $g$ proves our result.

The statement that for those $n$ for which $N\left(f^{n}\right) \neq 0 \quad\left|f \operatorname{xix}\left(g^{n}\right)\right|=$ $N\left(f^{n}\right)$ follows from the Nielsen number product theorem for FadellHusseini fibrations as well as the fact that our deformations of $\bar{f}$ and the appropriate maps on fibers are chosen to satisfy these criteria.

Deforming the periodic fibers of $u f v$. Let the distinct points $y_{0}, \ldots$, $y_{p-1} \in \operatorname{per}(\bar{u} \bar{f} \bar{v})$ be a typical orbit with $T_{y_{i}}=\pi^{-1}\left(y_{i}\right)$ and $(u f v)_{y_{i}}=$ $(u f v) \mid T_{y_{i}}: T_{y_{i}} \rightarrow T_{y_{i+1}}(i+1$ being calculated modulo $p)$. The orientability of the Fadell-Husseini fibration guarantees for $n=1,2, \ldots$ that

$$
L\left(\left[(u f v)^{p n}\right]_{0}\right)=L\left(\left[(u f v)^{p n}\right]_{y_{0}}\right)=L\left(\left[(u f v)_{y_{p-1}} \cdots(u f v)_{y_{0}}\right]^{n}\right) .
$$


Since

$$
\begin{aligned}
L\left(f^{n}\right)=L\left((u f v)^{n}\right) & =L\left((\bar{u} \bar{f} \bar{v})^{n}\right) L\left(\left[(u f v)^{n}\right]_{0}\right) \\
& =\left[\prod_{i=1}^{s}\left(1-\beta_{i}^{n}\right)\right]\left[\prod_{j=1}^{m}\left(1-\alpha_{j}^{n}\right)\right]
\end{aligned}
$$

is bounded as a function of $n$ and $L\left((\bar{u} \bar{f} \bar{v})^{n}\right)=\prod_{i=1}^{s}\left(1-\beta_{i}^{n}\right)=$ $L\left(\bar{f}^{n}\right) \not \equiv 0$, Lemma 1.3 guarantees us that either $\forall j\left|\alpha_{j}\right| \leq 1$ or some $\alpha_{j}=1$ so $\left\{L\left(\left[(u f v)^{p n}\right]_{y_{0}}\right)\right\}_{n=1}^{\infty}$ is bounded.

The deformation of the various $(u f v)_{y_{i}}$ is done in two stages. First we must show how to assume without loss that each such map and hence the composition $\left[(u f v)^{p}\right]_{y_{0}}=(u f v)_{y_{p-1}} \ldots(u f v)_{y_{0}}: T_{y_{0}} \rightarrow T_{y_{0}}$ is induced by a homomorphism. Then we can apply Theorem 1.5 to get a deformant $h_{y_{0}}$ on $T_{y_{0}}$ (which we extend to a deformant $h$ on $M^{m}$ which is the identity on all other periodic fibers of $u f v$ ) for which the composition $\left[(h u f v)^{p}\right]_{y_{0}}=h_{y_{0}}(u f v)_{y_{p-1}} \cdots(u f v)_{y_{0}}$ has finitely many periodic points. Then Lemma 2.5 applies to show that each $\left[(h u f v)^{p}\right]_{y_{i}}=(u f v)_{y_{i-1}} \cdots(u f v)_{y_{0}} h_{y_{0}}(u f v)_{y_{p-1}} \cdots(u f v)_{y_{i}}$ has finitely many periodic points. We then repeat this procedure on the other periodic orbits of $\bar{u} \bar{f} \bar{v}$ to complete the proof.

In order to show that we can assume that each $(u f v)_{y_{i}}$ is induced by a homomorphism we will use the fact that each fiber translation in $M^{m}$ is a deformant and localize our analysis to a set of locally trivial product charts for a finite number of fibers in $M^{m}$.

For each $i \quad(0 \leq i<p)$ let $w_{i}, z_{i} \in B^{s}$ be such that $\bar{v}\left(y_{i}\right)=w_{i}$, $\bar{f}\left(w_{i}\right)=z_{i}$, and $\bar{u}\left(z_{i}\right)=y_{i+1}$. Define paths $\omega_{i}$ from $y_{i}$ to $w_{i}$ and $\beta_{i}$ from $z_{i}$ to $y_{i+1}$ by letting $\omega_{i}(t)=\bar{v}_{t}\left(y_{i}\right)$ and $\beta_{i}(t)=\bar{u}_{t}\left(z_{i}\right)$ where $\bar{v}_{t}: \mathrm{id}_{B} \sim^{h} \bar{v}$ and $\bar{u}_{t}: \mathrm{id}_{B} \sim^{h} \bar{u}$ are the stages in the deformants $\bar{u}$ and $\bar{v}$. The local triviality of the fibration means that we can find disjoint open neighborhoods about each $w_{i}, y_{i}$, and $z_{i}$ as well as $x_{0}=\bar{\Gamma} \in B^{s}$ so that the points in $M^{m}$ that lie above any of these points can be written in the form $\left(\xi_{i}, \delta\right)$ where $\xi_{i} \in\left\{w_{i}, y_{i}, z_{i}\right\}_{i=0}^{p-1} \cup\left\{x_{0}\right\}$ and $\delta \in T^{r}$.

Now in $M^{m}$ we can write that $f\left(w_{i}, \delta\right)=\left(z_{i}, \hat{f}\left(w_{i}, \delta\right)\right)$ for some function $\hat{f}:\left[\left\{w_{i}\right\}_{i=0}^{p-1} \cup\left\{x_{0}\right\}\right] \times T^{r} \rightarrow T^{r}$. As a function of $\delta \in T^{r}$, $\hat{f}\left(x_{0}, \delta\right) \stackrel{\text { def }}{=} f_{0}(\delta): T^{r} \rightarrow T^{r}$ is induced by a homomorphism since $f$ itself is induced by a homomorphism. For each $i$ we let $\alpha_{i}(t)$ be a path from $w_{i}$ to $x_{0}$. Then $\bar{f} \alpha_{i}(t)$ is a path from $z_{i}$ to $x_{0}$ and Lemma 2.3 justifies the following calculation (the $\gamma_{\omega}$ translation maps are induced from the $\tau_{\omega}$, on the principal fiber through the local 
trivialization identifications just indicated):

$$
\begin{aligned}
\left(x_{0}, \gamma_{\bar{f} \alpha_{i}} \hat{f}\left(w_{i}, \delta\right)\right) & =\tau_{\bar{f} \alpha_{i}}\left(z_{i}, \hat{f}\left(w_{i}, \delta\right)\right)=\tau_{\bar{f} \alpha_{i}} f\left(w_{i}, \delta\right) \\
& =f \tau_{\alpha_{i}}\left(w_{i}, \delta\right)=f\left(x_{0}, \gamma_{\alpha_{i}}(\delta)\right) \\
& =\left(x_{0}, \hat{f}\left(x_{0}, \gamma_{\alpha_{i}}(\delta)\right)\right) .
\end{aligned}
$$

So $\gamma_{\bar{f} \alpha_{i}} \hat{f}\left(w_{i}, \delta\right)=\hat{f}\left(x_{0}, \gamma_{\alpha_{i}}(\delta)\right)$. Since the $\gamma$ translations are all deformants, we have the following chain of deformant equivalences: (as maps of $T^{r}$ )

$$
\hat{f}\left(w_{i}, \delta\right) \sim_{D} \gamma_{\bar{f}_{\alpha_{i}}} \hat{f}\left(w_{i}, \delta\right)=\hat{f}\left(x_{0}, \gamma_{\alpha_{i}}(\delta)\right) \sim_{D} \hat{f}\left(x_{0}, \delta\right) .
$$

Thus finally, we conclude

$$
\begin{aligned}
u f v\left(y_{i}, \delta\right) & =u f \tau_{\omega_{i}}\left(y_{i}, \delta\right)=u f\left(w_{i}, \gamma_{w_{i}}(\delta)\right) \\
& =u\left(z_{i}, \hat{f}\left(w_{i}, \gamma_{\omega_{i}}(\delta)\right)\right)=\tau_{\beta_{i}}\left(z_{i}, \hat{f}\left(w_{i}, \gamma_{\omega_{i}}(\delta)\right)\right) \\
& =\left(y_{i+1}, \gamma_{\beta_{i}} \hat{f}\left(w_{i}, \gamma_{\omega_{i}}(\delta)\right)\right) \sim_{D}\left(y_{i+1}, \hat{f}\left(w_{i}, \delta\right)\right) \\
& \sim_{D}\left(y_{i+1}, \hat{f}\left(x_{0}, \delta\right)\right)
\end{aligned}
$$

and so the required maps between fibers are indeed deformant equivalent to homomorphism induced maps as needed.

Case 2. $\left\{L\left(\bar{f}^{n}\right)\right\}_{n=1}^{\infty}$ unbounded. If $\left\{L\left(\bar{f}^{n}\right)\right\}_{n=1}^{\infty}$ is unbounded then by Lemma 1.3 since $\left\{L\left(f^{n}\right)\right\}_{n=1}^{\infty}$ is bounded it must mean that $L\left(f_{0}^{n}\right)$ $\equiv 0 \equiv L\left(f^{n}\right)$ so we must deform $f$ to be periodic point free.

The deformation, (actually a left deformant homotopy), involves applying the Anosov trick (Theorem 2.4) with an appropriately chosen element $Y \in \widetilde{\mathscr{G}}$ to each iterate of $f$. Because $L\left(f_{0}\right)=\operatorname{det}\left(\dot{F}_{0}-I\right)=$ $\operatorname{det}(\dot{F}-I)=0$ there is a Jordan chain $\mathscr{K}=\left\{Y_{0}, \ldots, Y_{l}\right\}$ associated to the eigenvector 1 of $\dot{F}$ in which $Y_{0} \in \tilde{\mathscr{G}}$. We note that the entire chain, and hence also $Y_{l}$, lies in $\widetilde{\mathscr{G}}$. This follows by induction since if $Y_{s} \in \widetilde{\mathscr{G}}$ but $Y_{s+1} \notin \widetilde{\mathscr{G}}$ then there would be a nontrivial kernel to $\dot{\bar{F}}-I$ forcing $L(\bar{f})=0$ and so $\left\{L\left(\overline{f^{n}}\right)\right\}_{n=1}^{\infty}=\{0\}$ would not be unbounded as we have assumed. We let $Y=Y_{l}$.

Consider the iterates of $\exp (t Y) f$ on $M^{m}$. We see that by an inductive calculation (using the fact that $f$ is induced by the homomorphism $F$ ) that

$$
\begin{aligned}
(\exp (t Y) f)^{n}(x) & =\exp (t Y) \exp (t \dot{F} Y) \exp \left(t \dot{F}^{2} Y\right) \cdots \exp \left(t \dot{F}^{n-1} Y\right) f^{n}(x) \\
& =\exp \left(t\left(Y+\dot{F} Y+\dot{F}^{2} Y+\cdots+\dot{F}^{n-1} Y\right)\right) f^{n}(x)
\end{aligned}
$$


The last equality uses that all the $\hat{F}^{i} Y$ belong to $\widetilde{\mathscr{G}} \subseteq \operatorname{Center}(\mathscr{G})$.

We claim that for each $n$ the vector $W_{n}=Y+\dot{F} Y+\cdots+F^{n-1} Y$ does not belong to $\left(\dot{F}^{n}-I\right)(\mathscr{G})$. Then the Anosov trick applies to each iterate of $f$ and we have that for each $n$, the set

$$
\mathscr{A}_{n}=\left\{t: \operatorname{fix}\left(\exp \left(t W_{n}\right) f^{n}\right)=\operatorname{fix}\left((\exp (t Y) f)^{n}\right) \neq \varnothing\right\}
$$

is countable. Hence $\bigcup_{n=1}^{\infty} \mathscr{A}_{n}$ is countable and a $t_{0}$ must exist so that $\operatorname{per}\left(\exp \left(t_{0} Y\right) f\right)=\varnothing$ and we are done. (Notice that translation of $f$ by $\exp \left(t_{0} Y\right) \sim \exp (0 Y)=1_{G}$ has the desired left deformation homotopy form we predicted.)

To prove the claim we note that $W_{n} \subseteq \operatorname{span}(\mathscr{K})$ and so by the Jordan decomposition of $\mathscr{G}$ into a collection of invariant subspaces formed by the span of the various Jordan chains for $F$ the result will follow once we see that each $W_{n}$ has a nonzero component in the direction of $Y_{l}$. This is true by an easy calculation which shows that $W_{n}-n Y_{l} \subseteq \operatorname{span}\left(\left\{Y_{1}, \ldots, Y_{l-1}\right\}\right)$.

3. Solvmanifolds. We now embark on the next level of generalization of nilmanifolds, the solvmanifolds. We will see that in analogy to our previous work, there is a fibration for solvmanifolds (called the Mostow fibration) which allows us to view a solvmanifold as being composed of a nilmanifold and a torus. These fibrations allow us to proceed in roughly the same way as we did for the nilmanifolds. There is a principal difference, however, in that these fibrations in general are nonorientable and hence have no Lefschetz number or Nielsen number product theorem. This leads to a number of interesting complications to our theory as well as some open questions for future study.

A solvmanifold $R^{m}$ is a compact smooth transitive $G$-space where $G$ is a solvable Lie group. The following characterization of solvmanifolds was first discovered by Mostow [17].

THeOrem 3.1. Let $R^{m}$ be a solvmanifold. Then $R^{m}$ is equivariantly homeomorphic to a coset space of the form $J / \Delta$ where $J$ is a connected, simply connected solvable Lie group (homeomorphic to some finite dimensional euclidean space), and $\Delta$ is a closed uniform subgroup.

The principal difference seen here, between a solvmanifold and a nilmanifold besides the solvability criterion for $J$, is the fact that we cannot assume that $\Delta$ is discrete. We can only say that $\Delta$ has no 
subgroup normal in $J$ which is not discrete. $R^{m}$ is called a special solvmanifold when $\Delta$ can be taken discrete in Mostow's representation. Since nilpotent groups are solvable, it is clear that all nilmanifolds are special solvmanifolds. After presenting a general theorem for all solvmanifolds, we will specialize our study to a certain class of low dimensional special solvmanifolds.

Using Mostow's representation we suppose that $J$ is a connected, simply connected, solvable Lie group and we let $\Delta_{0}$ be the connected component of $\Delta$ which contains the identity. By analyzing the Lie algebra $\mathscr{T}$ for $J$ and the fact that it imbeds in some finite dimensional solvable matrix Lie algebra of upper triangular matrices it is possible to show that $[\mathscr{J}, \mathscr{J}]$ and hence also $[J, J]$ is nilpotent. Thus there is a unique maximal connected, simply connected nilpotent subgroup $K$ of $J$ which contains both $[J, J]$ and $\Delta_{0}$ as normal subgroups. We let $\mathscr{N}_{J}$ denote the set of all those connected simply connected subgroups of $K$ which contain both $[J, J]$ and $\Delta_{0}$. With $\widetilde{M}_{J}$ the minimal element of $\mathscr{N}_{J}$ the following can be proved.

Theorem $3.2[16,17]$ (Mostow fibration). Suppose $R^{m}=J / \Delta$ as in Theorem 3.1 is a solvmanifold. Then to each subgroup $H$ of $J$ belonging to $\mathscr{N}_{J}$ there is a Mostow fibration for $R^{m}$ given by $\Delta H / \Delta=$ $M^{s} \hookrightarrow J / \Delta=R^{m} \rightarrow J / \Delta H=T^{r}$ where $M^{s}$ is a nilmanifold and $T^{r}$ a torus. Furthermore, for any $f: R^{m} \rightarrow R^{m}$ we can, up to homotopy, replace $f$ by a fiber preserving map of the Mostow fibration for $R^{m}$ corresponding to $\widetilde{M}_{J}$. The homotopy type of the base map $\bar{f}: T^{r} \rightarrow T^{r}$ obtained in this way is unique.

We call the Mostow fibration for $R^{m}$ corresponding to $\widetilde{M}_{J}$ the minimal Mostow fibration for $R^{m}$.

Unlike the Fadell-Husseini fibration for nilmanifolds, McCord [16] has observed that the Mostow fibrations of a non-nilmanifold solvmanifold are never orientable. For a study of periodic points this means that in general for a Mostow fiber preserving map $f: R^{m} \rightarrow R^{m}$ on a solvmanifold the self maps on fibers over different fixed points of $\bar{f}$ can have widely varying Lefschetz numbers and in general there is no Lefschetz number or Nielsen number product theorem. Thus the relationship between the sequences of Nielsen or Lefschetz numbers of the iterates of $\bar{f}, f$, and the maps $\left\{f_{x}^{n}: x \in \mathrm{fix}\left(\bar{f}^{n}\right)\right\}$ is much more complicated than before. In order to begin to obtain an understanding of these relationships, we must first understand the relationship between the Nielsen classes of $f, \bar{f}$ and $f_{x}$ for $x \in \operatorname{fix}(\bar{f})$. Suppose 
that $x \in \operatorname{fix}(\bar{f})$. Then McCord [16] observed that in a Mostow fibration for points $y, z \in M_{x}^{s}$ we have that $y \sim_{f_{x}} z$ iff $y \sim_{f} z$. This allows one to prove

COROLlaRY 3.3. Suppose $f: R^{m} \rightarrow R^{m}$ is a fiber preserving map of a Mostow fibration for $R^{m}$ with base map $\bar{f}: T^{r} \rightarrow T^{r}$. If $\left\{x_{1}\right\}, \ldots$, $\left\{x_{p}\right\}$ are distinct singleton essential Nielsen classes of $\bar{f}$ then the essential Nielsen classes of each $f_{x_{i}}: M_{x_{i}}^{s} \rightarrow M_{x_{i}}^{s}$ form a subset of the essential Nielsen classes of $f$ so $\sum_{i=1}^{p} N\left(f_{x_{i}}\right) \leq N(f)$.

McCord's [16] observations were used to prove that for maps $f: R^{m} \rightarrow R^{m}$ on solvmanifolds $|L(f)| \leq N(f)$. When $\left\{N\left(f^{n}\right)\right\}_{n=1}^{\infty}$ is unbounded, $\operatorname{per}(f)$ cannot be homotopically finite. Unlike the situation for nilmanifolds, there are examples (e.g. see Halpern's discussion of the Klein bottle [9]) of cases on solvmanifolds where $\left\{L\left(f^{n}\right)\right\}_{n=1}^{\infty}$ is bounded but $\left\{N\left(f^{n}\right)\right\}_{n=1}^{\infty}$ is not. Thus here we will conduct an examination of what circumstances, besides the boundedness of $\left\{N\left(f^{n}\right)\right\}_{n=1}^{\infty}$, are needed to assure that $\operatorname{per}(f)$ is indeed homotopically finite.

Homotopically finite periodicity. From Theorem 3.2 for a self map $f$ on a solvmanifold the sequences $\left\{N\left(f^{n}\right)\right\}_{n=1}^{\infty}$ and $\left\{N\left(\bar{f}^{n}\right)\right\}_{n=1}^{\infty}$ are both homotopy invariants of $f$. In analogy with a portion of our proof for nilmanifolds we present a general result for all solvmanifolds that depicts when the boundedness of both $\left\{N\left(f^{n}\right)\right\}_{n=1}^{\infty}$ and $\left\{N\left(\bar{f}^{n}\right)\right\}_{n=1}^{\infty}$ imply that $\operatorname{per}(f)$ is homotopically finite. For this work we will call on the definitions and insight obtained in $\S 1$ regarding the discussion for the absence of inessential periodic points from Halpern's deformations on tori.

As before, we will have need to deform various maps between fibers of a fiber preserving $f: R^{m} \rightarrow R^{m}$. Since Mostow fibrations are nonorientable the fiber translation functions are no longer necessarily deformants. The next result circumvents this difficulty. The proof is an easy consequence of a theorem in [1].

CoRollary 3.4. Suppose that $f: E \rightarrow E$ is a fiber preserving map of the fibration $F \hookrightarrow E \rightarrow B$ covering $\bar{f}: B \rightarrow B$. Further, assume that $y_{0}, \ldots, y_{q}$ are distinct points of $B$ and that $g_{0}, \ldots, g_{q}$ are maps such that, for each $i f_{y_{i}}: F_{y_{i}} \rightarrow F_{\bar{f}\left(y_{i}\right)}$ is homotopic to $g_{i}$. Let $W \subset E$ be a closed set not containing any $y_{i}$. Then there is a fiber preserving homotopy $H_{t}: f \sim g$ which is constant on $W$, has constant projection, and restricts to a homotopy $f_{y_{i}} \sim g_{i}$ over $y_{i}$. 
THEOREM 3.5. Let $f: R^{m} \rightarrow R^{m}$ be a fiber preserving map on the minimal Mostow fibration for $R^{m}$ covering $\bar{f}$. Suppose that both $\left\{N\left(f^{n}\right)\right\}_{n=1}^{\infty}$ and $\left\{N\left(\bar{f}^{n}\right)\right\}_{n=1}^{\infty}$ are bounded. If the $\bar{g} \sim \bar{f}$ obtained by applying Theorem 1.5 to $\bar{f}$ has no inessential periodic points, then $\operatorname{per}(f)$ is homotopically finite.

Proof. We first use the existing homotopy of $\bar{f}$ to $\bar{g}$ and lift this to a fiberwise homotopy of $f$. Without loss then we can assume that $f$ covers $\bar{g}$ and has a finite number of periodic fibers. Consider a periodic orbit $y_{0}, \ldots, y_{p-1}$ of distinct periodic points of $\bar{g}$ with $\bar{g}\left(y_{i}\right)=y_{i+1} \quad(i+1$ calculated modulo $p)$. The proof for this orbit consists of two major steps. First, we must show that the composition $\left(f^{p}\right)_{y_{0}}=f_{y_{p-1}} \cdots f_{y_{0}}: M_{y_{0}}^{s} \rightarrow M_{y_{0}}^{s}$ forms, with its iterates, a bounded sequence of Nielsen (or Lefschetz) numbers. Then we can use Theorem 2.6 to perform the individual fiberwise homotopies and accomplish the result.

To prove that the iterates of $\left(f^{p}\right)_{y_{0}}$ give a sequence of bounded Nielsen numbers we use the fact that $\bar{g}$ has no inessential periodic points. Hence since each $y_{i}$ has minimal period $p$ under $\bar{g}$, $L\left(\bar{g}^{p}\right)=\prod_{i=1}^{k}\left(1-\beta_{i}^{p}\right) \neq 0$. Thus no $\beta_{i}^{p}$ is 1 . By assumption $\left\{\left|\prod_{i=1}^{k}\left(1-\beta_{i}^{p n}\right)\right|\right\}_{n=1}^{\infty}=\left\{N\left(\bar{g}^{p n}\right)\right\}_{n=1}^{\infty}$ is bounded so $\forall i \quad\left|\beta_{i}^{p}\right| \leq 1$. From Theorem 1.5 whenever $0 \neq\left|L\left(\bar{g}^{p n}\right)\right|=N\left(\bar{g}^{p n}\right)=\left|\operatorname{fix}\left(\bar{g}^{p n}\right)\right|$ it means that each $y_{i}$ is its own essential Nielsen class for $\bar{g}^{p n}$. Thus by Corollary 3.3, the values of $n$ for which $L\left(\bar{g}^{p n}\right) \neq 0$ give $N\left(\left(f^{p}\right)_{y_{0}}^{n}\right) \leq$ $N\left(f^{p n}\right)$. Thus the boundedness of $\left\{N\left(f^{n}\right)\right\}_{n=1}^{\infty}$ implies that, for these $n, N\left(\left(f^{p}\right)_{y_{0}}^{n}\right)$ is bounded. Thus if $L\left(\left(f^{p}\right)_{y_{0}}^{n}\right)=\prod_{j=1}^{m}\left(1-\alpha_{j}^{n}\right)$ then it must mean that as a function of $n \prod_{i=1}^{k}\left(1-\beta_{i}^{p n}\right) \prod_{j=1}^{m}\left(1-\alpha_{j}^{n}\right)$ is bounded. (This product is zero for those $n$ with $L\left(\bar{g}^{p n}\right)=0$.) Since no $\beta_{i}^{p}=1$ Lemma 1.3 implies that either some $\alpha_{j}=1$ or else all the $\alpha_{j}$ satisfy $\left|\alpha_{j}\right| \leq 1$. Either way, we can conclude that $\left\{N\left(\left(f^{p}\right)_{y_{0}}^{n}\right)\right\}_{n=1}^{\infty}$ is bounded as needed.

We can now use Corollary 3.4 to deform $f$ in such a way that each $f_{y_{i}}$ is homotopied, with the correct identifications, to be induced by a homomorphism. The deformation has $\bar{g}$ as a constant projection throughout so no new periodic fibers are introduced during the homotopy. We can also use Corollary 3.4 to guarantee that the homotopy is constant in an open neighborhood of all other periodic fibers besides the $M_{y_{i}}^{s}$ thus assuring that no previously performed fiber homotopies are disturbed during the process. Thus the composition $\left(f^{p}\right)_{y_{0}}: M_{y_{0}}^{s} \rightarrow M_{y_{0}}^{s}$ will be induced by a homomorphism. Then by 
using Theorem 2.6 we can find deformants $u_{0}$ and $v_{0}$ on $M_{y_{0}}^{s}$ so that $\operatorname{per}\left(\left(u_{0} f^{p} v_{0}\right)_{y_{0}}\right)$ will be finite. We then extend $u_{0}$ and $v_{0}$ to deformants $u$ and $v$ on $R^{m}$ over $\mathrm{id}_{T^{r}}$ which are equal to the identity on all other fibers over points of $\operatorname{per}(\bar{g})$. Lemma 2.5 then guarantees that the other $\operatorname{per}\left(\left(u_{0} f^{p} v_{0}\right)_{y_{i}}\right)$ are finite as well.

REMARKS. (1) Note that the use of deformants on the $\left(f^{p}\right)_{y_{0}}$ is essential here since this map in general is a composition of fiber maps of differing homotopy types and our proof requires us to minimize periodic points by deforming $f$ and hence these individual fiber maps in the composition $\left(f^{p}\right)_{y_{0}}$. However, in the original deformation of $\bar{f}$ to $\bar{g}$ we do not need a deformant homotopy. As long as we guarantee that $\bar{g}$ satisfies the two conditions that whenever $L\left(\bar{g}^{n}\right) \neq 0$ then each element of $\operatorname{fix}\left(\bar{g}^{n}\right)$ belongs to its own essential Nielsen class for $\bar{g}^{n}$ and $\bar{g}$ has no inessential periodic points, the proof will work. This paves the way for any future improvements of Halpern's methods which might show that inessential periodic points can be eliminated.

(2) By Corollary 1.8 we know that if $\operatorname{dim} T^{r}=r=1$ or 2 then it is enough to just have $\left\{N\left(f^{n}\right)\right\}_{n=1}^{\infty}$ and $\left\{N\left(\bar{f}^{n}\right)\right\}_{n=1}^{\infty}$ bounded since the no inessential periodic points criterion for $\bar{g}$ will be satisfed. The analysis at the close of $\S 1$ indicates how we might determine for other tori and $\bar{f}$ whether this condition can be easily met.

(3) From Theorem 3.2 any $f: R^{m} \rightarrow R^{m}$ is, up to homotopy, fiber preserving on the minimal Mostow fibration for $R^{m}$. If it happens that $f$ is homotopic to a fiber preserving map of some other Mostow fibration for $R^{m}$ besides the minimal one, and the resulting base map $\hat{\bar{f}}$ in this situation satisfies the hypotheses required on $\bar{f}$, especially the inessential periodic point condition, then the same proof will show that $\operatorname{per}(f)$ is homotopically finite. Since the other Mostow fibrations for $R^{m}$, in general, have a smaller dimensional torus in the base than the minimal Mostow fibration and $\hat{\bar{f}}$ is induced by a restriction of the linear map inducing $\bar{f}$, this observation could conceivably provide a proof in some instances where the original hypotheses fail.

(4) If $\left\{N\left(\bar{f}^{n}\right)\right\}_{n=1}^{\infty}=\{0\}$ then we can choose $\bar{g}$ with $\operatorname{per}(\bar{g})=\varnothing$ thus showing that $\operatorname{per}(f)$ is homotopically empty. We will also be able to remove all the periodic points of $f$ if every appropriate iterate of the maps between fibers over periodic points of $\bar{g}$ has Lefschetz number zero.

(5) We cannot assume that $N\left(f^{n}\right)=\mid$ fix $\left(g^{n}\right) \mid$ whenever $N\left(f^{n}\right) \neq$ 0 . The problem is that for a given $n$ we may have $N\left(\bar{g}^{n}\right) \neq 0$ but 
there may be $x \in \operatorname{fix}\left(\bar{g}^{n}\right)$ for which $N\left(\left(g^{n}\right)_{x}\right)=0$ but $\operatorname{fix}\left(\left(g^{n}\right)_{x}\right) \neq$ $\varnothing$. (If then there are $\left(g^{n}\right)_{y}$ for other $y \in \mathrm{fix}\left(\bar{g}^{n}\right)$ with nonzero Nielsen numbers then $N\left(g^{n}\right)=N\left(f^{n}\right)$ could be nonzero.)

(6) Notice that we cannot in general expect that $g \sim_{D} f$. The problem is that since Mostow fibrations are nonorientable, not every fiber translation map is a deformant. Thus it may not be possible to deform each $f_{y_{i}}$ to be homomorphism induced by using only deformant homotopies. (See the proof of Theorem 2.6.)

3.1. An important class of solvmanifolds. In the last section we proved that a map $f: R^{m} \rightarrow R^{m}$ has $\operatorname{per}(f)$ homotopically finite provided that both $\left\{N\left(f^{n}\right)\right\}_{n=1}^{\infty}$ and $\left\{N\left(\bar{f}^{n}\right)\right\}_{n=1}^{\infty}$ are bounded and that $\bar{f}$ can be deformed to have a finite number of periodic points with the property that each occurs, at its minimal iterate, as a singleton essential Nielsen class. These, more or less, are conditions that allow us to mimic a portion of our proof in the nilmanifold case. Now we would like to undertake a deeper study of how the boundedness of $\left\{N\left(f^{n}\right)\right\}_{n=1}^{\infty}$ affects the boundedness of $\left\{N\left(\bar{f}^{n}\right)\right\}_{n=1}^{\infty}$. In the nilmanifold situation you will recall that $\left\{N\left(f^{n}\right)\right\}_{n=1}^{\infty}$ bounded implies that either $\left\{N\left(\bar{f}^{n}\right)\right\}_{n=1}^{\infty}$ is bounded or else $\left\{N\left(\bar{f}^{n}\right)\right\}_{n=1}^{\infty}$ is unbounded and $\left\{N\left(f^{n}\right)\right\}_{n=1}^{\infty}=\{0\}$. The second case, by orientability, is equivalent to the fact that the appropriate iterates of the maps $\left\{f_{x}^{n}:\{x\}\right.$ is an essential Nielsen class of $\left.\bar{f}^{n}\right\}$ all have Nielsen number zero. Because the Mostow fibrations of a non-nilmanifold solvmanifold are nonorientable, so these maps in general have different Nielsen numbers, which will thus often be nonzero, we would not expect this second possibility to occur in the solvmanifold situation. Thus we wonder if the boundedness of $\left\{N\left(f^{n}\right)\right\}_{n=1}^{\infty}$ does not, in at least some solvmanifolds, imply that $\left\{N\left(\bar{f}^{n}\right)\right\}_{n=1}^{\infty}$ must also be bounded. A first step in justifying this intuition is a deeper study of how the maps on the fibers $\left\{f_{x}: x \in T^{r}\right\}$ are related.

LEMMA 3.6. Suppose that $F \hookrightarrow E \stackrel{\pi}{\rightarrow} B$ is a Hurewicz fibration of compact manifolds and $f: E \rightarrow E$ is a fiber-preserving map over $\bar{f}$. Let $x_{0} \in B$ be a basepoint for $B$ and assume that $\omega$ is a path starting at some $x \in B$ and ending at $x_{0}$. Then $f_{x}: F_{x} \rightarrow F_{\bar{f}(x)}$ is homotopic to the composition $\tau_{\bar{f} \omega^{-1}} f_{x_{0}} \tau_{\omega}$.

In the case of a Mostow fibration, the coset structure of our path lifting function easily gives the following. 
LEMMA 3.7. Let $\omega: I \rightarrow J$ induce a path, also called $\omega$, in $T^{r}=$ $J / \Delta H$. If $g \in J$ is such that $\pi(g \Delta)=\omega(0) \Delta H$ then $\tau_{\omega}(g \Delta)=$ $\omega(1) \omega(0)^{-1} g \Delta$.

In order to apply Lemma 3.6 to our periodic point problem we now present a model for the specific structure of a broad class of solvmanifolds. It is a fact which we will shortly discuss, that the class of all special solvmanifolds that arise from $\mathbf{R}^{3}$ (equipped with some solvable non-nilpotent Lie group structure) are all members of this class. This will eventually allow us to see that in these dimension 3 examples the boundedness of $\left\{N\left(f^{n}\right)\right\}_{n=1}^{\infty}$ is, alone, enough to guarantee that $\operatorname{per}(f)$ is homotopically finite.

We will say that the solvmanifold $R^{m}=J / \Delta$ is a special $N B A$ solvmanifold (i.e. is nilpotent by abelian) provided that $J$ is the semidirect product $J=G \times{ }_{\varphi} \mathbf{R}^{r}$ where $G \approx \mathbf{R}^{s}$ is a nilpotent Lie group and $\mathbf{R}^{r}$ is the standard abelian euclidean space Lie group formed by componentwise addition. Thus the group operation for $J$ is defined in terms of the operations for $G, \mathbf{R}^{r}$ and $\varphi: \mathbf{R}^{r} \rightarrow \operatorname{Aut}(G)$ by $(x, a)(y, b)=\left(x \varphi_{a}(y), a+b\right)$. We also require that $\Delta=\Gamma \times_{\tilde{\varphi}} \mathbf{Z}^{r}$ where $\Gamma$ is a uniform discrete subgroup of $G$ and $\tilde{\varphi}$ is induced from $\varphi$ by restriction.

Since $[J, J] \subseteq G \times_{\varphi}\{0\} \cong G, R^{m}$ will have a Mostow fibration $M^{s}=G / \Gamma \hookrightarrow J / \Delta \rightarrow \mathbf{R}^{r} / \mathbf{Z}^{r}=J / \Delta G$. We will call this the canonical Mostow fibration for $R^{m}$ with respect to the decomposition in the definition of a special NBA solvmanifold. Now if $G$ is such that this is the minimal Mostow fibration for $R^{m}$, i.e. if $G=[J, J]$, then any self map on $R^{m}$ will be homotopic to a fiber preserving map of this fibration and we can consider our periodic point question for all such self maps. Otherwise, for this model, we must restrict ourselves to maps $f: R^{m} \rightarrow R^{m}$ which satisfy $f_{\sharp}\left(\pi_{1}\left(M^{s}\right)\right) \subseteq \pi_{1}\left(M^{s}\right)$. By identifying $\pi_{1}\left(M^{s}\right)$ with $\Gamma$ we see that this means that if $f$ is induced by a homomorphism $F: J \rightarrow J$, then we must have $F(\Gamma) \subseteq$ $\Gamma$.

Let $R^{m}$ be an arbitrary special NBA solvmanifold and suppose that $f: R^{m} \rightarrow R^{m}$ is a fiber preserving map of the canonical Mostow fibration for $R^{m}$ covering $\bar{f}: T^{r} \rightarrow T^{r}$. We would like now to compute a suitable description of the composition in Lemma 3.6 for the case when $x \in \operatorname{fix}(\bar{f})$. Since $\varphi$ is a homomorphism $\varphi_{\alpha}^{-1}=\varphi_{-\alpha}$. Since $\pi((w, \alpha) \Delta)=(w, \alpha) \Delta G=(1, \alpha) \Delta G$ we can use $[\alpha]$ to depict the coset $(w, \alpha) \Delta G$ in $T^{r} . M_{\alpha}^{s}$ will then be used for $M_{[\alpha]}^{s}$ and $f_{\alpha}$ for 
$f_{[\alpha]}$. We denote the coset $(w, \alpha) \Delta$ of $R^{m}$ by $\langle w\rangle_{\alpha}$ so $\pi\left(\langle w\rangle_{\alpha}\right)=[\alpha]$. We let [0] be the basepoint of $T^{r}$ so that

$$
M^{s}=M_{0}^{s}=\pi^{-1}([0])=\left\{\langle w\rangle_{0}: w \in G\right\}=G / \Gamma .
$$

The next lemma gives us an obvious and important way to equate the fundamental groups of the various $M_{\alpha}^{s}$.

LEMMA 3.8. $\langle w\rangle_{\alpha}=\langle 0\rangle_{\alpha} \Leftrightarrow \varphi_{-\alpha}(w) \in \Gamma$ so that the fundamental group of each $M_{\alpha}^{s}$ can be identified with $\Gamma$ after a rescaling by $\varphi_{-\alpha}$.

With the help of Lemma 3.6 we can now perform a computation on how the Lefschetz numbers of different maps on fibers over points of $\operatorname{fix}(\bar{f})$ vary. Without loss in generality to the homotopy type of $f$, we assume that $\bar{f}: T^{r} \rightarrow T^{r}$ is induced by a linear map $\Theta: \mathbf{R}^{r} \rightarrow \mathbf{R}^{r}$. Thus [0] $\in \operatorname{fix}(\bar{f})$. We let $F_{0}: G \rightarrow G$ be the homomorphism inducing a map on $M_{0}^{s}$ in the homotopy class of $f_{0}: M_{0}^{s} \rightarrow M_{0}^{s}$. Then the Lie algebra of $G$ is, as a vector space, isomorphic to $\mathbf{R}^{s}$ and the derivative of $F_{0}, F_{0}: \mathbf{R}^{s} \rightarrow \mathbf{R}^{s}$, is a Lie algebra homomorphism. The correspondence assigning to $\alpha \in \mathbf{R}^{r}$ the derivative of $\varphi_{\alpha}$, which we denote by $\Phi_{\alpha}$, is a homomorphism $\Phi: \mathbf{R}^{r} \rightarrow \mathrm{Gl}_{s}(\mathbf{R})$. Lemma 3.7 easily gives

LemMA 3.9. Suppose $[\alpha] \in \operatorname{fix}(\bar{f})$. Let $\omega$ be a path in $T^{r}$ from $[\alpha]$ to [0] induced by a path, also called $\omega$, in $J$ of the form $(1, \omega(t))$ from $(1, \alpha)$ to the identity $(1,0)$. Then a path lifting function can be chosen so that $\tau_{\omega}$ amounts to left translation in $J$ by $(1,-\alpha)$. Hence $\tau_{\omega}\left(\langle x\rangle_{\alpha}\right)=\left\langle\varphi_{-\alpha}(x)\right\rangle_{0}$. Similarly, $\tau_{\bar{f} \omega^{-1}}\left(\langle y\rangle_{0}\right)=\left\langle\varphi_{\Theta \alpha}(y)\right\rangle_{\alpha}$.

The following is our desired Lefschetz number calculation. In it we let $I$ stand for the $r \times r$ identity matrix. The proof uses Lemmas 3.6, 3.8, and 3.9 along with the standard properties for determinants.

LEMMA 3.10. If $[\alpha] \in \operatorname{fix}(\bar{f})$ then $f_{\alpha}: M_{\alpha}^{s} \rightarrow M_{\alpha}^{s}$ has Lefschetz number

$$
L\left(f_{\alpha}\right)=\operatorname{det}\left[\left(\Phi_{(2 I-\Theta) \alpha}-\dot{F}_{0}\right) \Phi_{(\Theta-2 I) \alpha}\right] .
$$

Thus if $\left[\alpha_{1}\right], \ldots,\left[\alpha_{p}\right]$ are distinct fixed points of $\bar{f}$ and $L(\bar{f})=$ $\operatorname{det}(\Theta-I) \neq 0$ then $f$ has an essential Nielsen class contained in each $M_{\alpha_{t}}^{s}$, provided that

$$
\forall i \quad \operatorname{det}\left[\Phi_{(2 I-\Theta) \alpha_{i}}-\dot{F}_{0}\right] \neq 0 .
$$

In this case $N(f) \geq p$. 
3.2. 3-dimensional special solvmanifolds. Let $J \approx \mathbf{R}^{3}$ be a connected, simply connected solvable Lie group and $\Delta \subset J$ a discrete uniform subgroup. It is our goal in this section to study our periodic point question for spaces of the form $R^{3}=J / \Delta$. Of course if $J$ is nilpotent then $R^{3}$ is a nilmanifold and Theorem 2.6 tells us all we currently want to know. Thus we will assume throughout that $J$ is solvable but non-nilpotent. Appropriately, we begin our study with a discussion of some results in [4] that tell us what kind of manifolds arise in this fashion.

TheOREM 3.11 [4]. Suppose $R^{3}=J / \Delta$ is the coset space of a nonnilpotent solvable Lie group $J \approx \mathbf{R}^{3}$ and a uniform discrete subgroup $\Delta$. Then $J$ is isomorphic to a semidirect product of abelian factors $\mathbf{R}^{2} \times_{\varphi} \mathbf{R}$ for some one parameter subgroup $\varphi$ of $\mathrm{Sl} 2(\mathbf{R})$. Up to a similarity transformation of $\varphi$ and possible additional equivariant rescaling homeomorphism we can assume that $\varphi(t)$ is either the diagonal matrix $\varphi_{t}^{\xi}=\operatorname{diag}\left(e^{\xi t}, e^{-\xi t}\right)$ (we call this exponential with real parameter $\xi$ ) or

$$
\varphi_{t}^{\xi}=\left[\begin{array}{cc}
\cos (2 \pi \xi t) & \sin (2 \pi \xi t) \\
-\sin (2 \pi \xi t) & \cos (2 \pi \xi t)
\end{array}\right]
$$

(We call this rotational with real parameter $\xi$.) In the case of the exponential Lie group, which gives rise to what we call an exponential solvmanifold, the parameter $\xi$ is such that $2 \neq e^{\xi}+e^{-\xi} \in \mathbf{Z}$. In the case of the rotational group, which gives rise to what we call a rotational solvmanifold, the parameter $\xi=\frac{n}{p}$ where $n \in \mathbf{Z}$ and $p \in$ $\{1,2,3,4,6\}$. The only and hence the minimal Mostow fibration for these solvmanifolds fibers $R^{3}$ over a circle with fiber $T^{2}$. Thus, up to homotopy, we can always assume that any self map $f: R^{3} \rightarrow R^{3}$ is a fiber-preserving map of this fibration and thus covers a self map $\bar{f}$ on the circle.

We are now ready to present our principal result for iterates on these solvmanifolds. The next lemma will allow us to remove the extra hypotheses needed to prove the general result of Theorem 3.5.

LEMMA 3.12. Suppose that $f: R^{3} \rightarrow R^{3}$ is a fiber preserving map of the Mostow fibration for a rotational or exponential solvmanifold (as in Theorem 3.11). If $\left\{N\left(f^{n}\right)\right\}_{n=1}^{\infty}$ is bounded, then so is $\left\{N\left(\bar{f}^{n}\right)\right\}_{n=1}^{\infty}$. 
Proof. Suppose that $\left\{N\left(\bar{f}^{n}\right)\right\}_{n=1}^{\infty}$ is unbounded. It will suffice to prove that $\left\{N\left(f^{n}\right)\right\}_{n=1}^{\infty}$ must also be unbounded. Thus $m=|\operatorname{deg}(\bar{f})|$ $>1$. Without loss we may assume that $\operatorname{deg}(\bar{f})>0$ since if this is not the case we can replace $f$ by $f^{2}$ (and hence $\bar{f}$ by $\bar{f}^{2}$ ) to make this so.

The basic idea of the proof is that for each positive integer $q$ we must find an $n$ so that Lemma 3.10 may be applied to $f^{n}$ and $\bar{f}^{n}$ to conclude that $N\left(f^{n}\right) \geq q$. Throughout our argument we will assume that $\bar{f}$ is induced by a homomorphism. This way, we will know exactly what the fixed points of each $\bar{f}^{n}$ are. In applying this corollary we note that for our three dimensional solvmanifolds since each automorphism $\varphi_{t}$ is linear, it follows that $\Phi_{t}$ always has the same matrix representation as $\varphi_{t}$. Replacing $\Theta$ by multiplication by $m^{n}$ we let $\Lambda_{n}(\alpha)=\operatorname{det}\left(\Phi_{\left(2-m^{n}\right) \alpha}-F_{0}^{n}\right)$ so that by Lemma 3.10 for each $\alpha \in[0,1)$ satisfying $\bar{f}^{n}([\alpha])=[\alpha]$ (i.e. $\left(m^{n}-1\right) \alpha \in \mathbf{Z}$ ) we have that $N\left(\left(f^{n}\right)_{\alpha}\right) \neq 0$ iff $\Lambda_{n}(\alpha) \neq 0$. For $n=1,2, \ldots$ let

$$
\dot{F}_{0}^{n}=\left[\begin{array}{ll}
a_{n} & b_{n} \\
c_{n} & d_{n}
\end{array}\right] .
$$

For the exponential solvmanifold of parameter $\xi$

$$
\Lambda_{n}(\alpha)=\operatorname{det} \dot{F}_{0}^{n}+1-a_{n} e^{-\xi\left(2-m^{n}\right) \alpha}-d_{n} e^{\xi\left(2-m^{n}\right) \alpha} .
$$

Now we observe that, for each $n, \Lambda_{n}(\alpha)$ is either constantly 0 as a function of $\alpha$ or else is zero for at most two values of $\alpha$. (This can be verified by checking the number of zeroes of the derivative (w.r.t. $\alpha$ ) of each $\Lambda_{n}(\alpha)$.) Since for a given $n, \bar{f}^{n}$ has $m^{n}-1$ fixed points and this is unbounded with increasing $n$, our result for the exponential solvmanifolds will be verified once we check that it cannot be the case that for some $M>0$ we have that $\forall n \geq M \Lambda_{n}(\alpha) \equiv 0$. [I.e. we must prove that there are infinitely many $n$ with $\Lambda_{n}(\alpha) \not \equiv 0$ so that for each of these $n$ at least $m^{n}-3$ of the fixed points of $\bar{f}^{n}$ are suitable for use in Corollary 3.10.] We perform this check by noting that if $\Lambda_{n}(\alpha) \equiv 0 \equiv \Lambda_{2 n}(\alpha)$ then $a_{n}=d_{n}=0=a_{2 n}=d_{2 n}$ so $\dot{F}_{0}^{2 n}=0$ and thus $\Lambda_{2 n}(\alpha) \equiv 1$ (a contradiction).

For a rotational solvmanifold of parameter $\xi$

$$
\begin{aligned}
\Lambda_{n}(\alpha)= & \operatorname{det} \dot{F}_{0}^{n}+1-\left(a_{n}+d_{n}\right) \cos \left[2 \pi \xi\left(2-m^{n}\right) \alpha\right] \\
& +\left(c_{n}-b_{n}\right) \sin \left[2 \pi \xi\left(2-m^{n}\right) \alpha\right] .
\end{aligned}
$$

Thus if $\Lambda_{n}(\alpha) \equiv 0 \equiv \Lambda_{2 n}(\alpha)$ then $a_{n}+d_{n}=a_{2 n}+d_{2 n}=0$ and $c_{n}-b_{n}=0=c_{2 n}-b_{2 n}$ so $\dot{F}_{0}^{2 n}=0$ and $\Lambda_{2 n}(\alpha) \equiv 1$ as before. 
Thus $\Lambda_{n}(\alpha) \not \equiv 0$ for infinitely many $n$. For such $n$ we can via some trigonometric addition formulas show that for some numbers $\beta$ and $\gamma$

$$
\Lambda_{n}(\alpha)=0 \Leftrightarrow \cos \left[2 \pi \xi\left(2-m^{n}\right) \alpha+\gamma\right]=\beta
$$

so the zeros of $\Lambda_{n}$ occur for

$$
\alpha=\frac{ \pm \cos ^{-1}(\beta)-\gamma}{2 \pi \xi\left(2-m^{n}\right)}+\frac{k}{\xi\left(2-m^{n}\right)}
$$

with $k \in \mathbf{Z}$. Thus the zeros of $\Lambda_{n}(\alpha)$ come in two sets according to whether we choose the + or - above. The elements of each set are spaced by a distance of $\frac{1}{\xi\left(m^{n}-2\right)}$. The fixed points of $\bar{f}$ for $\alpha \in[0,1)$ occur with a spacing of $\frac{1}{m^{n}-1}$. Thus we can see that even if only every $j$ th $(j=1,2, \ldots)$ fixed point of $\bar{f}$ is a root of $\Lambda_{n}(\alpha)=0$ from one of the two sets, then we must have $\frac{j}{m^{n}-1}=\frac{d}{\xi\left(m^{n}-2\right)}$ for some $d \in \mathbf{Z}$. But $d=\frac{j \xi\left(m^{n}-2\right)}{m^{n}-1}$ is an integer for at most finitely many values of $n$. Arguing this way for both sets we see that for any small $\varepsilon>0$ we can say that for all but a finite number of the $n$ for which $\Lambda_{n}(\alpha) \not \equiv 0$ less than $\varepsilon$ percent of the fixed points of $\bar{f}$ are roots of $\Lambda_{n}(\alpha)=0$. Thus as we consider increasing $n$ it is clear that

$$
\mid\left\{\alpha \in[0,1):[\alpha] \in \operatorname{fix}\left(\bar{f}^{n}\right) \text { and } \Lambda_{n}(\alpha) \neq 0\right\} \mid
$$

can be as large as desired.

Thus our results on nilmanifolds and tori (see Corollary 1.8) along with Theorems 3.11 and 3.5 give the following.

Corollary 3.13. Suppose $J$ is a connected, simply connected solvable Lie group of dimension 3 or less and $\Delta \subset J$ is a uniform discrete subgroup. Suppose $f: R^{3}=J / \Delta \rightarrow R^{3}$. Then $\operatorname{per}(f)$ is homotopically finite iff $\left\{N\left(f^{n}\right)\right\}_{n=1}^{\infty}$ is bounded.

The Klein bottle, a nonspecial solvmanifold. In order to demonstrate the extent of our techniques, we close the paper by considering a new proof of known results for the Klein bottle.

Let $J=\mathbf{C} \times{ }_{\varphi} \mathbf{R}$ with $\varphi(t)(z)=e^{2 \pi i t} z$ and $\Delta=\left\{\left(n+i y, \frac{m}{2}\right) \mid n, m \in\right.$ $\mathbf{Z}, y \in \mathbf{R}\}$. In [3] it is observed that $K=J / \Delta$ is homeomorphic to the Klein bottle and that $K$ cannot be represented as a special solvmanifold. However, other than the fact that $\Delta$ has the form $\Delta=$ $\Gamma \times{ }_{\tilde{\varphi}} \mathbf{Z}^{r}$ with $\Gamma$ not discrete, the form for $K$ is like our model for NBA solvmanifolds. The nondiscrete nature of $\Delta$ gives us a discrete group 
of fiber translation maps (in this case $Z_{2}$ ) and we are able to prove the needed relationship between the Nielsen numbers of the base and fiber and obtain an alternate proof of the following fact already known from [9].

THEOREM 3.14. Suppose that $f: K \rightarrow K$ is a map in which $\left\{N\left(f^{n}\right)\right\}_{n=1}^{\infty}$ is bounded. Then there is a $g \sim f$ for which $\operatorname{per}(g)$ is finite.

Proof. With the above description of $K$ it is not hard to determine the following facts:

1. The only Mostow fibration for $K$ corresponds to the abelian subgroup $H=\mathrm{C}$ of $J$ which fibers $K$ over the circle $S^{1}$ with fibers $S^{1}$.

2. By Theorem 3.5 and Remark 2 following that theorem it will suffice for us to show that if $f: K \rightarrow K$ is fiber preserving over $\bar{f}$ then when $\left\{N\left(\bar{f}^{n}\right)\right\}_{n=1}^{\infty}$ is unbounded then the same must be true of $\left\{N\left(f^{n}\right)\right\}_{n=1}^{\infty}$.

3. The base circle can be parametrized by elements of the form $[\alpha]=\left\{\left(0, \alpha+\frac{n}{2}\right): n \in \mathbf{Z}\right\}$.

4. With this representation the fiber over $[\alpha] S_{\alpha}^{1}$ has the form $\{(w, \alpha): w \in \mathbf{C}\} / \sim$ where $\left(w_{1}, \alpha\right) \sim\left(w_{2}, \alpha\right)$ iff the real parts of $w_{1}$ and $w_{2}$ differ by an integer.

5. Suppose $\bar{f}$ is multiplication by $d>1$. (If $\operatorname{deg}(\bar{f})<-1$ just replace $f$ by $f^{2}$.) Choose $\alpha \in\left[0, \frac{1}{2}\right)$ with $d \alpha \equiv \alpha\left(\bmod \frac{1}{2}\right)$ and let $\omega$ be the path in $\left[0, \frac{1}{2}\right)$ from $\alpha$ to 0 . Then from Lemma $3.7 \omega$ induces paths $\omega$ and $\bar{f} \omega^{-1}$ in the base and fiber translation maps $\tau_{\omega}: S_{\alpha}^{1} \rightarrow S_{0}^{1}$ given by $\tau_{\omega}(w, \alpha)=(w, 0)$ and $\tau_{\bar{f} \omega^{-1}}: S_{0}^{1} \rightarrow S_{d \alpha}^{1}$ given by $\tau_{\bar{f} \omega^{-1}}(w, \alpha)=( \pm w, d \alpha)$ with the + or - depending on whether $2(d-1) \alpha \in \mathbf{Z}$ is even or odd, respectively.

6. The identification of $S_{d \alpha}^{1}$ with $S_{\alpha}^{1}$ by right multiplication by $(0,(1-d) \alpha) \in \Delta$ carries $( \pm w, d \alpha)$ to $( \pm w, \alpha)$. Therefore, for a given degree $d$ for $\bar{f}, \operatorname{deg}\left(f_{\alpha}\right)= \pm \operatorname{deg}\left(f_{0}\right)$ for $f_{\alpha}$ on $S_{\alpha}^{1}$ with the + or - depending on whether $\alpha=\frac{i}{2(d-1)} 0 \leq i<d-1$ has even or odd $i$, respectively.

7. To consider iterates of $f$ we replace $d$ by $d^{l} l=1,2, \ldots$ and note that $\left|\operatorname{fix}\left(\bar{f}^{l}\right)\right|$ is unbounded. Now if $\operatorname{deg}\left(f_{0}\right)=0$, then $\forall l \operatorname{deg}\left(f_{0}^{l}\right)=0$ so all the $N\left(\left(f^{l}\right)_{\alpha}\right)$ for $\alpha \in \operatorname{fix}\left(\bar{f}^{l}\right)$ will contribute +1 to $N\left(f^{l}\right)$ so this will grow without bound as $l$ increases. A similar unboundedness will of course be true if $\left|\operatorname{deg}\left(f_{0}\right)\right|>1$. Finally, if 
$\operatorname{deg}\left(f_{0}\right)= \pm 1$ then roughly half of the $N\left(\left(f^{l}\right)_{\alpha}\right)$ will contribute +2 to $N\left(f^{l}\right)$ so this will grow without bound as well.

\section{REFERENCES}

[1] G. Allaud and E. Fadell, A fiber homotopy extension theorem, Trans. Amer. Math. Soc., 104 (1962), 239-251.

[2] D. Anosov, The Nielsen number of maps of nilmanifolds, Russian Math. Surveys, 40 (1985), 149-150.

[3] L. Auslander, An exposition on the structure of solvmanifolds, Bull. Amer. Math. Soc., 79 (1973), 227-261.

[4] L. Auslander, L. Green and F. Hahn, Flows on Homogeneous Spaces, Ann. of Math. Stud., vol. 53, Princeton University Press (1963).

[5] R. Brooks, R. Brown, J. Pak and D. Taylor, Nielsen numbers of maps of tori, Proc. Amer. Math. Soc., 52 (1975), 398-400.

[6] R. F. Brown, The Lefschetz Fixed Point Theorem, Scott, Foresman and Company (1970).

[7] E. Fadell and S. Husseini, On a theorem of Anosov on Nielsen numbers for nilmanifolds, Nonlinear Functional Analysis and its Applications (Reidel), 173 (1986), 46-51.

[8] B. Halpern, Periodic points on tori, Pacific J. Math., 83 (1979), 117-133.

[9] _- Periodic points on the Klein bottle, never published.

[10] P. Heath, R. Piccinini and C. Y. You, Nielsen Type Numbers for Periodic Points, I, Proceedings from Tianjin 1988 (Topological Fixed Point Theory and Applications), Lecture Notes in Math., vol. 1411, Springer-Verlag, Berlin and New York, 1983, pp. 88-106.

[11] P. Heath and C. Y. You, Nielsen type numbers for periodic points II, preprint.

[12] B. Jiang, Lectures on Nielsen Fixed Point Theory, Contemp. Math., vol. 14, Amer. Math. Soc., Providence, RI, 1982.

[13] _ Fixed Point Classes From a Differentiable Viewpoint, Proceedings from Sherbrooke, Quèbec 1980 (Fixed Point Theory), Springer-Verlag Lecture Notes in Math., 886, 163-170.

[14] T. Kiang, The Theory of Fixed Point Classes, Springer-Verlag (1989).

[15] A. I. Mal'cev, On a Class of Homogeneous Spaces, English transl., Amer. Math. Soc. Transl. (1), 9 (1962), 276-307.

[16] C. McCord, Nielsen numbers and Lefschetz numbers on solvmanifolds, Pacific J. Math., 147 (1991), 153-164.

[17] G. D. Mostow, Factor spaces of solvable groups, Ann. of Math., 60 (1954), 1-27.

Received April 27, 1992 and in revised form December 10, 1992.

TEXAS A \& M University

College Station, TX 77843

Current address: University of Nevada, Reno

Reno, NV 89557

E-mail address: keppelma@math.unr.edu 


\section{PACIFIC JOURNAL OF MATHEMATICS}

Founded by

E. F. BeCKenbach (1906-1982) F. Wolf (1904-1989)

\section{EDITORS}

Sun-Yung A. Chang

(Managing Editor)

University of California

Los Angeles, CA 90024-1555

chang@math.ucla.edu

\section{F. Michael Christ}

University of California

Los Angeles, CA 90024-1555

christ@math.ucla.edu

\section{Herbert Clemens}

University of Utah

Salt Lake City, UT 84112

clemens@math.utah.edu

\author{
THOMAS ENRIGHT \\ University of California, San Diego \\ La Jolla, CA 92093 \\ tenright@ucsd.edu \\ Nicholas ERcolanI \\ University of Arizona \\ Tucson, AZ 85721 \\ ercolani@math.arizona.edu \\ R. FINN \\ Stanford University \\ Stanford, CA 94305 \\ finn@gauss.stanford.edu \\ VAUGHAN F. R. JONES \\ University of California \\ Berkeley, CA 94720 \\ vfr@math.berkeley.edu
}

Steven KerckhofF

Stanford University

Stanford, CA 94305

spk@gauss.stanford.edu

Martin SCHARLEMANN

University of California

Santa Barbara, CA 93106

mgscharl@math.ucsb.edu

Harold Stark

University of California, San Diego

La Jolla, CA 92093

V. S. VARADARAJAN

University of California

Los Angeles, CA 90024-1555

vsv@math.ucla.edu

\section{SUPPORTING INSTITUTIONS}

\author{
UNIVERSITY OF ARIZONA \\ UNIVERSITY OF BRITISH COLUMBIA \\ CALIFORNIA INSTITUTE OF TECHNOLOGY \\ UNIVERSITY OF CALIFORNIA \\ UNIVERSITY OF MONTANA \\ UNIVERSITY OF NEVADA, RENO \\ NEW MEXICO STATE UNIVERSITY \\ OREGON STATE UNIVERSITY
}

UNIVERSITY OF OREGON

UNIVERSITY OF SOUTHERN CALIFORNIA

STANFORD UNIVERSITY

UNIVERSITY OF HAWAII

UNIVERSITY OF UTAH

WASHINGTON STATE UNIVERSITY

UNIVERSITY OF WASHINGTON 


\section{PACIFIC JOURNAL OF MATHEMATICS}

Volume $164 \quad$ No. $1 \quad$ May 1994

Entropy versus orbit equivalence for minimal homeomorphisms

M. MiChaEL BoYle and DAVID E. HANDELMAN

The hyperspaces of infinite-dimensional compacta for covering and cohomological dimension are homeomorphic

TADEUSZ WLADYSLAW DOBROWOLSKI and LEONARD RUBIN

The index of transversally elliptic operators for locally free actions

JefFrey Stephen Fox and Peter Evarts Haskell

Unit indices of some imaginary composite quadratic fields

MiKiHITO HIRABAYASHI

Periodic points on nilmanifolds and solvmanifolds

EDWARD KEPPELMANN

Branched coverings of surfaces with ample cotangent bundle

MiCHAEL JEROME SPURR

Evolutionary existence proofs for the pendant drop and $n$-dimensional 147 catenary problems

ANDREW GUY STONE

Lattices of Lipschitz functions

NiKOLAI ISAAC WEAVER

Correction to: "Trace rings for verbally prime algebras" 